\title{
The ontogeny of the feature-positive effect in young chicks
}

\author{
JAMES S. MILLER \\ State University of New York, Binghamton, New York \\ and \\ SANDERS A. MCDOUGALL and JAMES F. ZOLMAN \\ University of Kentucky, Lexington, Kentucky
}

\begin{abstract}
The wide generality of the feature-positive effect (FPE) has caused speculation that the FPE may represent innate biases in the stimulus control of discriminative responding. There is little experimental evidence to date ragarding this possibility. In the present study, 1- or 4-day-old chicks were trained on a feature-positive (FP) or feature-negative (FN) discrimination with heat reinforcement. After the acquisition phase, these subjects received extinction training followed by a reacquisition phase. The FP performance was superior to the FN performance in both age groups. Extinction resulted in improved discrimination performance in both the FP and FN conditions. Unmasking of FN learning by the extinction treatment suggests that the FPE represents a deficit in performance, rather than an inability to learn the FN task. These data demonstrate that adult-like performance on feature discriminations is evident as early as the first day post-hatch.
\end{abstract}

Jenkins and Sainsbury $(1969,1970)$ found that the ability of pigeons to learn a visual discrimination between two stimulus displays was dependent on whether the distinguishing feature appeared on reinforced $(\mathrm{S}+$ ) or nonreinforced $(S-)$ trials. When the feature appeared on $S+$ trials (the feature-positive, or FP, condition), the discrimination was learned readily. However, when the feature appeared on S- trials (the feature-negative, or FN, condition), the discrimination was learned with difficulty, if at all. This superiority of FP performance relative to FN performance is referred to as the feature-positive effect (FPE). A more detailed analysis of response patterns during $\mathrm{S}+$ trials indicates that FP subjects respond directly at the feature, whereas FN subjects direct response toward background cues present on both $\mathrm{S}+$ and $\mathrm{S}-$ trials (referred to as the "common" element). Thus, this tendency to respond to or "track" the best predictor of reinforcement results in excellent FP discrimination performance and marginal FN performance.

Recent evidence (Hearst, 1984, 1987) indicates that subjects manifesting poor FN performance may have actually learned the discrimination. Following initial FN discrimination training, subjects were given sessions of extinction in which responding to $\mathrm{S}+$ or $\mathrm{S}-$ was not reinforced. Surprisingly, the subjects demonstrated a dramatic

This research was conducted at the University of Kentucky Medical Center. The authors would like to thank Eliot Hearst and D. F. McCoy for their valuable comments on an earlier version of the manuscript. We would also like to thank Joyce Jagielo for preparing the graphs and Jo Moore for typing the manuscript. Reprint requests should be sent to Jim Miller, Department of Psychology, State University of New York at Binghamton, Binghamton, NY 13901. improvement in discrimination performance beginning with the first session of extinction. On the basis of these data, Hearst concluded that the failure of FN subjects to respond differentially on $\mathbf{S}+$ and $\mathbf{S}$ - trials represented a deficit in performance, rather than an inability to learn the FN discrimination.

The FPE has been established as a general phenomenon, having been demonstrated in appetitive and aversive situations, and with Pavlovian and operant procedures (see Hearst, 1978, 1984, for reviews). The FPE has also been established with a variety of responses and reinforcers, and across a wide variety of subject populations, including rats (Reberg \& LeClerc, 1977), pigeons (Jenkins \& Sainsbury, 1969, 1970), monkeys (McCoy \& Yanko, 1983; Pace, McCoy, \& Nallan, 1980), human children (Sainsbury, 1971), and adults (Newman, Wolff, \& Hearst, 1980). The wide generality of the phenomenon, as well as the demonstration of the FPE in humans, has caused speculation that the FPE may not reflect learned response tendencies. Rather, the FPE is viewed as a reflection of an innate bias to process information based on the presence (but not the absence) of stimuli occurring on reinforced trials (Newman et al., 1980). There is little experimental evidence to date regarding this possibility.

Although all young animals are not equally suitable for studying the ontogeny of learning, the precocial chick has the ability to perform a variety of adult-like responses immediately after hatching (Zolman \& McDougall, 1983). The performance of 4-day-old chicks is superior to that of 1-day-olds on a number of learning tasks, with the 4day-old chicks exhibiting response patterns similar to those obtained with adults of other species (Mattingly \& Zol- 
man, 1980). In the present study, 1- or 4-day-old chicks reared in isolation were given FP or FN discrimination training. Demonstration of the FPE in 1-day-old subjects would provide support for the view that the FPE reflects innate biases in stimulus control. Inclusion of the 4-dayold subjects provided an assessment of the possibility that FP or FN discrimination performance may vary as a function of age.

\section{METHOD}

\section{Subjects and Rearing Procedures}

Twenty-four Colburn $\times$ Colburn chicks were incubated and hatched at $37^{\circ}-38^{\circ} \mathrm{C}$ and $58 \%-60 \%$ relative humidity. The chicks were removed from the dark hatching incubator within $4 \mathrm{~h}$ after hatching, banded, and then placed individually into white Plexiglas isolation tubes $(25 \mathrm{~cm}$ high $\times 20 \mathrm{~cm}$ in diameter $)$ in a temperature-controlled room set at $35^{\circ} \mathrm{C}$. For 4-day-old subjects, food and water were available ad lib until $15 \mathrm{~h}$ prior to testing, at which time food was removed. The breeder room was illuminated with fluorescent light from 6:00 a.m. until 11:00 p.m. daily.

\section{Apparatus}

Behavioral testing was performed in four conditioning chambers designed for testing young chicks with heat reinforcement (see Zolman, Pursley, Hall, \& Sahley, 1978). Each box was housed individually in a Forma Scientific incubator (Model 65) where the ambient temperature was set at $10^{\circ} \mathrm{C}$. Another Forma Scientific incubator with an auxiliary $2,000-\mathrm{W}$ heater was set at $35^{\circ} \mathrm{C}$, and plastic ductwork connected each cold incubator with this heat source. Heat onset in each chamber was controlled by solenoids whose activation displaced two circular butterfly valves. One valve instantaneously diverted the warm $35^{\circ}$ air up through the conditioning chamber, whereas the other valve opened to replace in the airflow system the same amount of warm air as that diverted. Illumination of a 28-V light (GE 1820) located under each conditioning chamber accompanied the presentation of the heat reinforcer. A white masking noise of $76 \mathrm{~dB}$ (re $20 \mu \mathrm{N} / \mathrm{m}^{2}$ ) was generated by a GrasonStadler white-noise generator (Model 901B) and delivered through a 10-cm speaker on the back wall of each conditioning incubator.

The response keys were mounted directly on IEE 12-unit in-line projectors that illuminated the transparent keys with the $\mathrm{S}+$ and S- stimuli. The presentation of stimulus events was programmed and controlled by a BRS/LVE Interact computer system.

\section{Procedure}

To establish keypeck responding, all subjects were given two sessions of discriminative autoshaping. Each session was made up of 24 trials ( 12 feature and 12 nonfeature). There was an interval of approximately $25 \mathrm{~min}$ between training sessions during which subjects were returned to the isolation tubes. A feature trial consisted of the illumination of a white dot on a red response key, whereas on nonfeature trials, the red response key alone was presented. All stimulus presentations were $16 \mathrm{sec}$ in duration and were separated by 5 -sec intertrial intervals (ITIs). Presentations of the S+ stimulus were followed by an 8 -sec presentation of forced air $\left(35^{\circ} \mathrm{C}\right)$ from beneath the chamber floor. If the subject pecked the key prior to the end of the stimulus presentation, heat reinforcement was delivered immediately, followed by the ITI. The S- stimulus was followed by only the ITI, regardless of whether a response occurred. The trials were presented randomly within the session, with the constraint that no more than three feature or nonfeature trials could occur successively. For feature-positive (FP) subjects ( $n=6 /$ age group), the white dot on the red keylight served as $\mathrm{S}+$, whereas the red key alone was S-. For feature-negative (FN) subjects ( $n=6 /$ age group), these contingencies were reversed.
Following autoshaping, the subjects were given 10 sessions of FP or FN discrimination training. The presentation of stimuli and the experimental parameters were identical to those used in the autoshaping sessions, except that if no response occurred on $\mathrm{S}+$ trials, reinforcement was not presented. Therefore, during acquisition training (Sessions 3-12), reinforcement was contingent on responding. During Sessions 13-16, all subjects were given extinction training in which responses to both $S+$ and $S-$ were nonreinforced. Following extinction training, the subjects were given four sessions of reacquisition training. As in original acquisition, reinforcement on $\mathrm{S}+$ trials was contingent on responding.

\section{RESULTS}

The primary dependent measure used for data analysis was the discrimination ratio (DR). The DR was calculated by dividing the number of $S+$ trials on which a response occurred by the total number of trials on which a response occurred. Thus, a DR of 1.0 would indicate perfect discrimination (all responses to $S+$ ), whereas a $\mathrm{DR}$ of .50 would indicate no preference for the $\mathrm{S}+$ or S- stimulus. Data were analyzed using a repeated measures analysis of variance (ANOVA) procedure. The ANOVAs were supplemented, when appropriate, by Newman-Keuls tests with the significance level set at $p<.05$.

There was no significant difference in discrimination performance due to age during acquisition. However, because a primary purpose of the study was to determine if the FPE was evident in 1-day-old subjects, the initial acquisition data for 1- and 4-day-old subjects were analyzed separately to determine if there was evidence of the FPE in each of the age groups. The mean discrimination ratios for the 1-day-old chicks are presented in Figure 1. An ANOVA performed on the acquisition data for 1-dayold subjects indicated that FP performance was superior to FN performance $[F(1,10)=5.96, p<.05]$. There was a significant effect of the session variable $[F(9,90)$ $=2.23, p<.05]$. The condition $\times$ session interaction was not significant. The mean discrimination ratios for the 4-day-old chicks are presented in Figure 2. Analysis of the acquisition data of the 4-day-old subjects yielded a similar pattern. FP performance was superior to FN per-

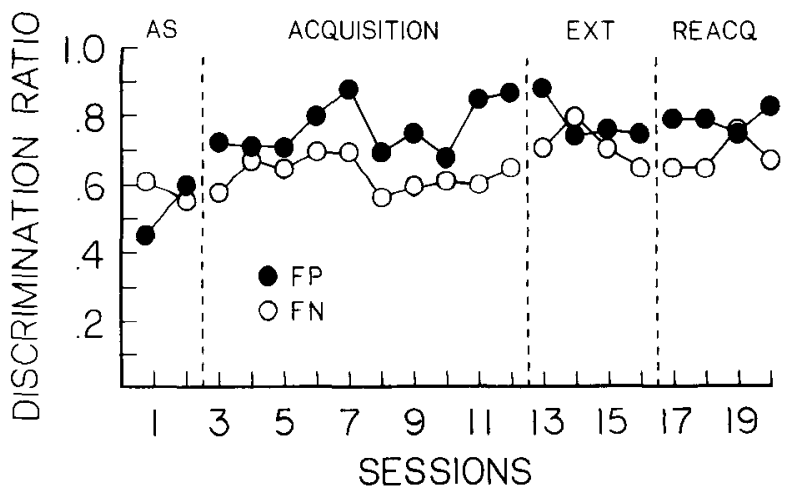

Figure 1. Mean discrimination ratios for 1-day-old chicks trained on a feature-positive (FP) or feature-negative (FN) discrimination. (AS: autoshaping; EXT: extinction; REACQ: reacquisition.) 


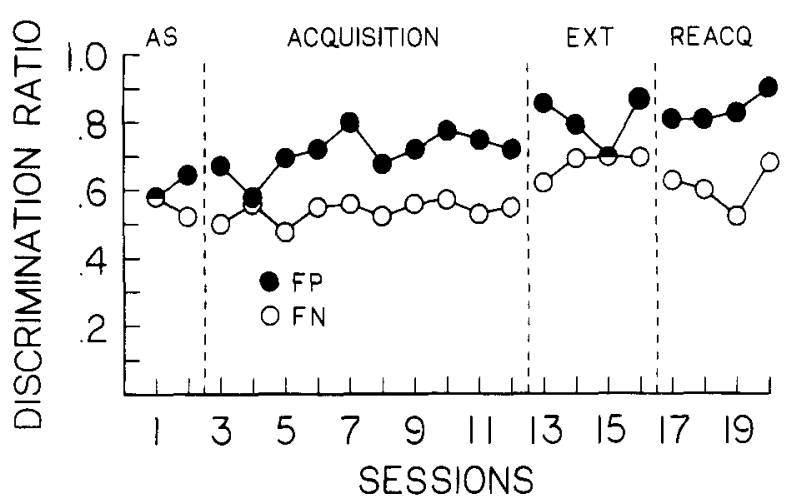

Figure 2. Mean discrimination ratios for 4-day-old chicks trained on a feature-positive (FP) or feature-negative (FN) discrimination. (AS: autoshaping; EXT: extinction; REACQ: reacquisition.)

formance $[F(1,10)=10.03, p<.01]$. There was a significant effect of the session variable $[F(9,90)=3.01$, $p<.01]$. The interaction was not significant.

The FPE occurs in adults because high rates of responding are suppressed on S- trials in the FP condition, but not in the FN condition. To determine if the FPE occurs in the 1- and 4-day-old chick for the same reason, the rate of responding to the $S-$ stimulus was examined during acquisition. The mean number of responses per session to $\mathrm{S}-$ by the treatment groups during acquisition are presented in Table 1. Analysis of the rate of responding to $S$ - indicated that subjects in the FP condition responded significantly less to the $\mathrm{S}-$ stimulus than did FN subjects in both the 1-day-old $[F(1,10)=16.92, p<.01]$ and 4-day-old $[F(1,10)=12.21, p<.01]$ conditions.

Data from the final acquisition and initial extinction session are presented in Table 2 . These data were analyzed to determine if extinction training resulted in an improvement in discrimination performance, which would be indicated by a significant effect of the session variable. Analysis of the data of FP subjects collapsed across age groups indicated a significant improvement in performance from the final acquisition session to the first extinction session $[F(1,10)=8.11, p<.05]$. This improvement in FP performance was evident only in 4-day-old subjects, as indicated by the significant age $\times$ session interaction $[F(1,10)=5.84, p<.01]$ and subsequent NewmanKeuls tests. The effect of age were not significant. Analysis of data from the FN subjects indicated a significant improvement in FN discrimination performance from the final acquisition session to the first extinction session $[F(1,10)=5.60, p<.05]$. The effect of age and the age $X$ session interaction were not significant.

An ANOVA performed on the reacquisition data indicated that FP performance was superior to FN performance $[F(1,20)=15.80, p<.01]$. The effects of age, sessions, and all interactions were not significant.

\section{DISCUSSION}

The present experiment examined the acquisition of feature discriminations by 1- and 4-day-old chicks. In both age groups, acquisition of the FP task was superior to that of the FN discrimination. The occurrence of the FPE in the 4-day-old chicks was not wholly unexpected, since these subjects have been shown to respond like adults on a variety of learning tasks (see Mattingly \& Zolman, 1980). However, contrary to previous research, which showed inferior performance by 1-day-old chicks, there were no significant differences in performance attributable to the age factor. The performance of the 4-day-old chicks was not superior to that of the 1-day-old subjects on either the FP or FN task. In fact, there was a trend toward better performance by the 1-day-old subjects. It may be that the heat served as a more effective reinforcer in these subjects, because they have a less well developed thermoregulatory system (Wekstein \& Zolman, 1967). Perhaps the result of greatest interest was the demonstration of the FPE in the 1-day-old subjects. This finding demonstrates that adult-like performance on feature discriminations is evident as early as the first day post-hatch.

The withdrawal of heat reinforcement during the extinction phase resulted in an immediate and significant improvement in FN discrimination performance in both age groups. Hearst (1987) reported a similar finding with pigeons using a food reinforcer. This unmasking of FN learning in extinction suggests that the FPE results from a deficit in performance, rather than from an inability to learn the FN discrimination. Hearst pointed out that this "extinction revelation" effect can be interpreted within the framework of a sign-tracking analysis of the FPE. This analysis suggests that extinction results in an attenuation of the predictability of the common or nonfeature stimulus. This change reveals control of responding by the inhibitory feature. In support of this sign-tracking analysis, unmasking of FN learning occurs with a variety of manipulations that degrade the information value of the common element (Hearst, 1987). However, subjects in the acquisition phase of the FP condition are already presumably responding to the best predictor of reinforcement. Therefore, unlike the FN situation, the improvement in FP discrimination performance during extinction

Table 1

Mean Number of Responses Per Session to S- by Feature-Positive (FP) and Feature-Negative (FN) 1- and 4-Day-Old Chicks During Acquisition

\begin{tabular}{ccc}
\hline Age & FP & FN \\
\hline 1 Day & 9.01 & 30.67 \\
4 Days & 16.10 & 49.51 \\
\hline
\end{tabular}

Table 2

Mean Discrimination Ratios for Feature-Positive (FP) and Feature-Negative (FN) 1- and 4-Day-Old Chicks During the Final Acquisition Session and First Extinction Session

\begin{tabular}{ccc}
\hline Condition & $\begin{array}{c}\text { Final } \\
\text { Acquisition } \\
\text { Session }\end{array}$ & $\begin{array}{c}\text { First } \\
\text { Extinction } \\
\text { Session }\end{array}$ \\
\hline FP-1 & .87 & .88 \\
FP-4 & .72 & .86 \\
FN-1 & .65 & .71 \\
FN-4 & .55 & .62
\end{tabular}


cannot be explained by a sign-tracking analysis via the revelation of "behaviorally silent" associations. Rather, these data suggest that the extinction-revelation effect may be a more general phenomenon that is not limited to a particular discrimination task, species, or reinforcer.

\section{REFERENCES}

HEARST, E. (1978). Stimulus relationships and feature selection in learning and behavior. In S. Hulse, H. Fowler, \& W. K. Honig (Eds.), Cognitive processes in animal behavior (pp. 51-88). Hillsdale, $\mathrm{NJ}$ Erlbaum.

Hearst, E. (1984). Absence as information: Some implications for learning, performance, and representational processes. In $\mathrm{H}$. L. Roitblat, T. G. Bever, \& H. S. Terrace (Eds.), Animal cognition (pp. 311332). Hillsdale, NJ: Erlbaum.

HeArst, E. (1987). Extinction reveals stimulus control: Latent learning of feature-negative discriminations in pigeons. Journal of Experimental Psychology: Animal Behavior Processes, 13, 52-64.

JENIINS, H. M., \& SAINSBURY, R. S. (1969). The development of stimulus control through differential reinforcement. In N. J. Mackintosh \& W. K. Honig (Eds.), Fundamental issues in associative learning (pp. 123-161). Halifax, Nova Scotia: Dalhousie University Press.

JenKINS, H. M., \& SAINSBURY, R. S. (1970). Discrimination learning with the distinctive feature on positive or negative trials. In D. Mostofsky (Ed.), Attention: Contemporary theory and analysis (pp. 239-273). New York: Appleton-Century-Crofts.

Mattingly, B. A., \& Zolman, J. F. (1980). Ontogeny of passive avoidance learning in domestic chicks: Punishment of key-peck and running responses. Journal of Comparative \& Physiological Psychology, 94, 718-733.

McCoY, D. F., \& YANKo, D. (1983). Feature-positive and featurenegative learning maintained by negative reinforcement in rhesus monkeys. Animal Learning \& Behavior, 11, 460-464.

Newman, J., WolfF, W. T., \& Hearst, E. (1980). The feature-positive effect in adult human subjects. Journal of Experimental Psychology: Human Learning \& Memory, 6, 630-650.

Pace, G. M., McCoy, D. F., \& Nallan, G. B. (1980). Feature-positive and feature-negative learning in the rhesus monkey and pigeon. American Joumal of Psychology, 94, 409-427.

ReberG, D., \& LeClerC, R. A. (1977). A feature-positive effect in conditioned suppression. Animal Learning \& Behavior, 5, 143-147.

SAINSBURY, R. S. (1971). The "feature-positive effect" and simultaneous discrimination learning. Journal of Experimental Child Psychology, 11, 347-356.

Wekstein, D. R., \& Zolman, J. F. (1967). Homothermic development of the young chick. Proceedings of the Society of Experimental Biology \& Medicine, 125, 294-297.

Zolman, J. F., \& McDougall, S. A. (1983). Young precocial birds: Animal models for developmental neurobehavioral research. Bird Behaviour, 5, 31-58.

Zolman, J. F., Pursley, D. G., Hall, J. A., \& Sahley, C. L. (1978). Cholinergic involvement in inhibitory behavior of the young domestic chick. Behavioral Biology, 23, 415-432.

(Manuscript received May 28, 1987;

revision accepted for publication November 23, 1987.) 